\title{
Método ultrassônico para estimativa do módulo de elasticidade de madeiras de Pinus taeda tratadas termicamente
}

\author{
Ultrasonic method for estimating the modulus of elasticity of Pinus taeda thermally treated wood
}

\author{
André Luiz Missio ${ }^{I^{*}}$ Darci Alberto Gatto ${ }^{\mathrm{I}, \mathrm{II}}$ Karina Soares Modes $^{\mathrm{III}}$ Elio José Santini ${ }^{\mathrm{I} \text {,IV }}$ \\ Diego Martins Stangerlin ${ }^{\mathrm{V}}$ Leandro Calegari ${ }^{\mathrm{VI}}$
}

RESUMO

O trabalho teve por objetivo estimar o módulo de elasticidade estático da madeira termorretificada de Pinus taeda por meio do método não destrutivo ultrassônico. Foram confeccionados 93 corpos de prova das pranchas de pinus com dimensões de 2,5x2,5x41,0cm (espessura, largura e comprimento). Avaliaram-se corpos de prova submetidos a dois tratamentos térmicos denominados Combinação e Estufa, além daqueles sem tratamento térmico. No tratamento de combinação, as peças foram termorretificadas em autoclave a $130^{\circ} \mathrm{C} \pm 3{ }^{\circ} \mathrm{C}$ e pressão de $2 \mathrm{kgf} \mathrm{cm}^{-2}$, por 3 horas e, após um período de condicionamento, aquecidas em estufa elétrica a $160^{\circ} \mathrm{C} \pm 1^{\circ} \mathrm{C}$, pelo mesmo período. Já as peças destinadas apenas ao tratamento em estufa foram submetidas à última condição. Os modelos matemáticos criados apresentaram $r^{2}$ entre 0,48 e 0,66 , sendo significativos em $1 \%$ de probabilidade de erro. Dessa forma, com o aprimoramento de metodologias de ensaios não destrutivos ultrassônicos, a presente técnica pode ser útil e eficiente para estimar o módulo de elasticidade estático da madeira de Pinus taeda.

Palavras-chave: método não destrutivo, Loblolly pine, modificação térmica.

\section{ABSTRACT}

This study aimed to estimate the modulus of elasticity of Pinus taeda thermally modified wood by ultrasonic nondestructive method. To achieve this, 93 samples with dimensions $2.5 \times 2.5 \times 41.0 \mathrm{~cm}$ (thickness, width and length) from pine boards were prepared. The wood samples were thermally modified through two distinct treatments (Combination and Oven). The Combination treatment was performed in an autoclave at $130^{\circ} \mathrm{C} \pm 3^{\circ} \mathrm{C}$ and pressure of $2 \mathrm{kgf} \mathrm{cm}^{-2}$ for 3 hours. After a period of conditioning, the wood samples were heated in an electric oven $160^{\circ} \mathrm{C} \pm 1{ }^{\circ} \mathrm{C}$ for the same time. On the other hand, wood samples of the Oven treatment was modified with only last condition. The mathematical models showed $r^{2}$ between 0.48 and 0.66 and significance at $1 \%$ of probability of error. Thus, improvement of ultrasonic nondestructive tests shows that technique could be useful and efficient in order to estimate modulus of elasticity of Pinus taeda wood.

Key words: nondestructive method, Loblolly pine, thermal modification.

\section{INTRODUÇÃO}

A avaliação não destrutiva dos materiais é a ciência que visa a identificar suas propriedades físicas e mecânicas, sem alterar as características, utilização final e assim usar essas informações para tomada de decisão em relação às aplicações do material (ROSS et al., 1998). O campo de testes não destrutivos (NDT) e avaliação não destrutiva

IPrograma de Pós-graduação em Engenharia Florestal (PPGEF), Centro de Ciências Rurais (CCR), Universidade Federal de Santa Maria (UFSM), 97105-900, Santa Maria, RS, Brasil. E-mail: andreluizmissio@gmail.com. *Autor para correspondência.

"Curso de Engenharia Industrial Madeireira, Centro de Engenharias (CENG), Universidade Federal de Pelotas (UFPel), Pelotas, RS, Brasil. IIICurso de Engenharia Florestal, Departamento de Engenharia Florestal, Universidade Federal de Rondônia (UNIR), Rolim de Moura, RO, Brasil.

${ }^{\text {IV }}$ Curso de Engenharia Florestal, CCR, UFSM, Santa Maria, RS, Brasil.

VInstituto de Ciências Agrárias e Ambientais, Universidade Federal de Mato Grosso (UFMT), Sinop, MT, Brasil.

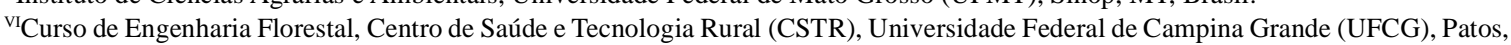
PB, Brasil. 
(NDE) dos materiais está em constante evolução, especialmente na área da madeira (BRASHAW et al., 2009), a qual, por ser um material anisotrópico e heterogêneo, mostra-se mais problemático (FEENEY et al., 1998).

De acordo com WANG et al. (2007), significativos esforços vêm sendo direcionados para o desenvolvimento de uma tecnologia consistente de NDE's, capaz de predizer com eficácia as propriedades intrínsecas da madeira, tanto para o controle de qualidade quanto para a classificação de produtos em linha de produção florestal. Dentre as tecnologias não destrutivas, pode-se citar o emprego do aparelho emissor de ondas de ultrassom.

A utilização do ultrassom consiste na emissão de pulsos elétricos por um circuito eletrônico. Esses pulsos elétricos são conduzidos por cabos coaxiais e convertidos em ondas elásticas pelo cristal piezelétrico, localizado nos transdutores. As vibrações mecânicas deslocam-se pelo material, que atenuam o sinal emitido pelo gerador. $\mathrm{O}$ sinal retardado é recuperado por outro cristal piezelétrico, sendo então amplificado e transformado em pulsos elétricos novamente para medir o tempo de propagação. Assim, tendo a distância e o tempo de percurso da onda, obtém-se a sua velocidade (CALEGARI, 2006).

Para a madeira, segundo BUCUR \& BÖNHKE (1994), os fatores que influenciam na propagação de ondas ultrassônicas são: características físicas, morfológicas (tipos de lenhos e ângulo de grã), presença de defeitos (nós e rachaduras), geometria das amostras, condições do meio (temperatura e umidade relativa) e procedimento utilizado para tomada das medidas (frequência e tipo de transdutor).

Dessa forma, a utilização da madeira em lugares e situações com determinadas restrições requer o estudo de melhorias de sua qualidade. Tratamentos térmicos reduzem a absorção de água (KARTAL et al., 2007), induzem alterações na coloração (ESTEVES et al., 2008a), reduzem a molhabilidade (HUANG et al., 2012) e melhoram a estabilidade dimensional (ESTEVES et al., 2007). Considerandose as muitas vantagens que o tratamento térmico traz para as propriedades da madeira, alguns processos já são utilizados em escala industrial em muitos países europeus (RODRIGUES, 2009).

Nesse contexto, este estudo objetivou determinar o módulo de elasticidade da madeira de Pinus taeda L., obtido por ensaio destrutivo de flexão estática através do método não destrutivo ultrassônico, após realização de diferentes tratamentos térmicos.

\section{MATERIAL E MÉTODOS}

As árvores de Pinus taeda foram obtidas de plantios homogêneos pertencentes à Fundação Estadual de Pesquisa Agropecuária - FEPAGRO FLORESTAS -, localizada em Boca do Monte, distrito de Santa Maria - RS. De forma aleatória, foram amostradas três árvores, com aproximadamente 25 anos de idade e, dessas, foram retiradas as duas primeiras toras com $2 \mathrm{~m}$ de comprimento, das quais se obtiveram duas pranchas de dimensões de 7,0x20,0cm (espessura x largura) diametralmente opostas, pelo método de desdobro tangencial, ao utilizar uma serra fita vertical.

$\mathrm{Na}$ confecção dos corpos de prova para os ensaios, tomou-se o cuidado de identificá-los com a numeração nas ripas, obtida no desdobro das pranchas e sequência de retirada nessa, de maneira a distribuir os corpos de prova adjacentes entre os tratamentos, evitando o efeito da posição diametral e longitudinal nos pranchões sobre os resultados. Foram confeccionados 93 corpos de prova com dimensões de 2,5x2,5x41,0cm (espessura, largura e comprimento) a partir das pranchas. Após essa etapa, o material permaneceu em câmara climatizada $\left(20^{\circ} \mathrm{C}\right.$ e $65 \%$ de UR) até alcançar umidade de equilíbrio próximo a $12 \%$.

Os corpos de prova foram submetidos a dois tratamentos de termorretificação: madeira tratada previamente em autoclave e, após um período de climatização, submetida ao tratamento em estufa (tratamento denominado Combinação, constituído por 32 corpos de prova) e madeira tratada somente em estufa (tratamento denominado Estufa, constituído por 31 corpos de prova), além da testemunha (madeira sem tratamento, constituído por 30 corpos de prova).

As fases, temperaturas e duração dos tratamentos de termorretificação foram em sequência: tratamento de termorretificação em autoclave à temperatura de $130^{\circ} \mathrm{C} \pm 3^{\circ} \mathrm{C}$ e pressão de $2 \mathrm{kgf} \mathrm{cm}^{-2}$ por três horas; período de condicionamento em câmara climatizada a $20^{\circ} \mathrm{C}$ e $65 \% \mathrm{UR}$ até massa constante; secagem prévia em estufa a $100^{\circ} \mathrm{C} \pm 1^{\circ} \mathrm{C}$ por 24 horas; tratamento de termorretificação em estufa elétrica a $160^{\circ} \mathrm{C} \pm 1^{\circ} \mathrm{C}$ por três horas. O tempo de duração dos tratamentos passou a ser contabilizado depois de atingida a temperatura requerida. A fase de secagem prévia foi realizada para redução do teor de umidade inicial dos corpos de prova antes do tratamento de termorretificação.

Após cada tratamento, foi calculada massa específica aparente na condição de câmara climatizada, $20^{\circ} \mathrm{C}$ e $65 \% \mathrm{UR}$, de acordo com a norma 
da Associação Brasileira de Normas Técnicas (NBR, 1997). Para o tratamento térmico do material, utilizouse uma autoclave de laboratório com dimensões internas aproximadas de 79x85x125cm (capacidade de 225 litros) e uma estufa elétrica, com circulação de ar e controle digital de temperatura.

Nos ensaios não destrutivos, o tempo de propagação da onda, em microssegundos $(\mu \mathrm{s})$, foi medido diretamente com auxílio de um equipamento de ultrassom. Os transdutores (sensores responsáveis pela emissão e recebimento das ondas) utilizados foram do tipo ponta seca com frequência de aproximadamente $54 \mathrm{kHz}$.

Foi necessária a calibração do aparelho emissor de ultrassom, que foi realizada sempre antes das análises por meio de um cilindro metálico, fornecido juntamente com o equipamento. Esse cilindro possui um tempo de propagação fixo $(20,5 \mu \mathrm{s})$. O processo de medição da velocidade de propagação (V) da onda foi composto de três medições realizadas nas extremidades das amostras, realizando um cálculo de média entre os três valores, assim podese calcular o módulo de elasticidade dinâmico (Ed), de acordo com a equação: $\mathrm{Ed}=\mathrm{V}^{2} \mathrm{x} \mathrm{ME} \times 10^{-6}$, em que: $\mathrm{Ed}=$ Módulo de elasticidade dinâmico $(\mathrm{MPa})$; $\mathrm{V}=$ Velocidade de propagação da onda $\left(\mathrm{m} \mathrm{s}^{-1}\right)$; $\mathrm{ME}=$ Massa específica da madeira na condição da câmara climatizada a $20^{\circ} \mathrm{C}$ e $65 \%$ de UR $\left(\mathrm{kg} \mathrm{m}^{-3}\right)$.

Posteriormente, os corpos de prova foram submetidos a ensaios destrutivos de flexão estática, para obtenção do módulo de elasticidade estático (Ec0). Para tanto, foi utilizada uma máquina universal de ensaios com capacidade de $200 \mathrm{kN}$, sendo que o ensaio prescreveu a norma da American Society for Testing and Materials - ASTM D143-94 (ASTM, 2000).

A análise estatística procedeu-se com um delineamento inteiramente casualizado, com uma espécie, três tratamentos (Combinação, Estufa e Testemunha) e trinta repetições (corpos de prova). Os resultados foram interpretados por meio de análise de variância (ANOVA) e criação de modelos matemáticos de regressão. Na ANOVA, analisouse o p-valor induzido pela estatística $\mathrm{F}$ em $5 \%$ de probabilidade de erro e, em caso de rejeição da hipótese nula $(\mathrm{P}<0,05)$, foi realizado o teste LSD (Least Significant Difference) de Fisher, em nível de $5 \%$ de probabilidade de erro para diferenciação da propriedade entre tratamentos.

Os modelos matemáticos de regressão linear foram selecionados através do coeficiente de determinação $\left(\mathrm{r}^{2}\right)$ do erro absoluto médio (EAM), estatística F e o $p$-valor. Para validação dos modelos, foi verificada a heterogeneidade de variância, normalidade e independência dos resíduos pelos testes White, Shapiro-Wilk e Durbin-Watson, respectivamente.

\section{RESULTADOS E DISCUSSÃO}

Os valores médios da ME dos corpos de prova que sofreram tratamento térmico Combinado (Autoclave + Estufa) apresentaram valores significativamente menores em relação aos que não sofreram tratamento térmico (Tabela 1). Essa

Tabela 1 - Valores de massa específica (ME), velocidade de propagação da onda ultrassônica (V) e módulo de elasticidade dinâmico (Ed) dos corpos de prova de Pinus taeda.

\begin{tabular}{|c|c|c|c|c|c|}
\hline & Tratamento & Máximo & Mínimo & Média & $\mathrm{CV}(\%)$ \\
\hline \multirow{3}{*}{$\operatorname{ME}\left(\mathrm{g} \mathrm{cm}^{-3}\right)$} & Combinação & 0,56 & 0,48 & $0,524 a$ & 4,70 \\
\hline & Estufa & 0,58 & 0,49 & $0,537 \mathrm{ab}$ & 3,67 \\
\hline & Testemunha & 0,58 & 0,49 & $0,543 b$ & 4,42 \\
\hline \multirow{3}{*}{$\mathrm{V}\left(\mathrm{m} \mathrm{s}^{-1}\right)$} & Combinação & 4340,00 & 3970,00 & $4185,42 b$ & 2,83 \\
\hline & Estufa & 4290,00 & 3910,00 & $4117,83 a$ & 2,61 \\
\hline & Testemunha & 4290,00 & 3930,00 & $4126,36 a b$ & 2,65 \\
\hline \multirow{3}{*}{$\mathrm{Ed}(\mathrm{MPa})$} & Combinação & 10554,10 & 7656,60 & $9198,62 a$ & 8,84 \\
\hline & Estufa & 10404,19 & 8165,62 & $9068,22 \mathrm{a}$ & 7,59 \\
\hline & Testemunha & 10410,70 & 7935,14 & $9253,20 \mathrm{a}$ & 7,60 \\
\hline
\end{tabular}

Em que: $\mathrm{ME}$ - massa especifica; $\mathrm{V}$ - velocidade de propagação da onda ultrassônica, Ed- módulo de elasticidade dinâmico; e CVcoeficiente de variação. Nas propriedades entre tratamentos, médias seguidas pela mesma letra na coluna não possuem diferença estatisticamente significativa em nivel de $95 \%$ de confiança, segundo teste LSD de Fisher.

Ciência Rural, v.43, n.4, abr 2013. 
diminuição se deve à degradação da hemicelulose, bem como à evaporação de extrativos que acontecem durante 0 tratamento térmico (ESTEVES \& PEREIRA, 2009). Os corpos de prova do tratamento denominado Estufa não apresentaram diferenças significativas em relação aos outros tratamentos.

A propriedade $\mathrm{V}$ apresentou diferenças significativas entre os tratamentos Combinação e Estufa, o que sugere que possíveis mudanças decorrentes de tratamentos térmicos a quente, como degradação das hemiceluloses (ESTEVES \& PEREIRA, 2009), podem influenciar na V. Os valores médios da $\mathrm{V}$ no sentido longitudinal, nos três tratamentos, foram semelhantes aos valores relatados por outros pesquisadores (CALEGARI et al., 2007; STANGERLIN et al., 2008a; STANGERLIN et al., 2008b), situando-se entre 4.000 e $6.000 \mathrm{~m} \mathrm{~s}^{-1}$.

Já os valores médios do $\mathrm{Ed}$ não apresentaram diferenças significativas entre os tratamentos. O Ed da espécie Eucalyptus grandis, de acordo com GARCIA et al. (2012), diminuiu em cerca de $13 \%$ após realização de tratamentos térmicos a $240^{\circ} \mathrm{C}$ por $4 \mathrm{~h}$. Sugere-se que tratamentos mais severos que os realizados no presente estudo possam causar maiores variações na estrutura anatômica da madeira e, consequentemente, afetar de forma mais significativa no Ed.

Com os resultados obtidos depois de realizado teste de médias (Tabela 2), nota-se que o maior valor médio do Ec0 foi encontrado no tratamento Combinado, mesmo que não se diferenciando significativamente dos outros tratamentos. ESTEVES et al. (2008b) também encontraram aumento no Ec0 após realização do tratamento Combinado.

Houve diferença significativa entre valores médios do Ec0 e do Ed nos tratamentos em que existiu

Tabela 2 - Valores máximos, mínimos e médios do módulo de elasticidade estático $(\mathrm{Ec} 0)$ dos corpos de prova de Pinus taeda, submetidos aos diferentes tratamentos.

\begin{tabular}{lcll}
\hline & & & \\
& & & \\
& Combinação & Estufa & Testemunha \\
Máximo & 15895,3 & 12509 & 11647,6 \\
Mínimo & 8047,19 & 6648,41 & 6879,94 \\
Média $(\mathrm{MPa})$ & $10335,0 \mathrm{a}$ & $9975,38 \mathrm{a}$ & $10200,0 \mathrm{a}$ \\
CV $(\%)$ & 11,62 & 11,24 & 9,22 \\
\hline
\end{tabular}

Em que: $\mathrm{CV}=$ coeficiente de variação em (\%). Entre a propriedade, médias seguidas pela mesma letra na linha não possuem diferença estatisticamente significativa em nível de $95 \%$ de confiança, segundo teste LSD de Fisher. termorretificação (Tabela 3). Já os corpos de prova que não realizaram termorretificação, denominados Testemunhas, não apresentaram diferenças significativas, baseado no teste de médias, o que mostra maior aproximação e precisão da metodologia entre o $\mathrm{Ed}$ e o $\mathrm{Ec0}$.

Em razão da natureza visco elástica da madeira, de acordo com OUIS (2002), quanto maior a frequência de excitação da fonte, maior é o valor inferido para o Ed. Segundo estudos realizados por BARTHOLOMEU (2001) e PUCCINI (2002), as relações entre $\mathrm{Ed} / \mathrm{Ec0}$ variam entre 1,06 e 1,38. Entretanto, ao analisar os resultados apresentados nas tabelas 1 e 2, verifica-se que os valores médios do Ed não foram superiores em relação ao $\mathrm{Ec0}$. Tal explicação pode estar relacionada com o tipo de onda gerado, provavelmente, do tipo superficial, que apresenta menor valor da $\mathrm{V}$, e consequente menor valor do Ed. NESVIJSKI (2003) afirma que alguns transdutores do tipo exponencial podem não gerar ondas direcionadas, o que proporciona maior tempo de propagação, quando comparado às ondas geradas por meio de transdutores de faces planas.

Para as estimativas do Ec0, nota-se que todos os modelos matemáticos propostos foram significativos em $1 \%$ de probabilidade de erro e atenderam aos testes de heterogeneidade de variância, normalidade e independência dos resíduos (Figura 1). Ao estudarem a mesma determinação na madeira de Pinus elliottii, STANGERLIN et al. (2008b) encontraram valores de coeficiente de determinação ajustado de 0,75 e 0,93 , para corpos de prova próximos à casca e à medula, respectivamente. As determinações encontradas foram superiores às do presente estudo.

Para madeira de Pinus taeda, BALLARIN \& NOGUEIRA (2005) observaram, para a mesma determinação, coeficiente de determinação de 0,89 (lenho juvenil), 0,94 (lenho adulto) e 0,89 (lenho juvenil e lenho adulto). As menores determinações encontradas no presente estudo podem ser explicadas pelas modificações anatômicas ocorridas na madeira após as termorretificações, já que, ao analisar essa propriedade, BOONSTRA et al. (2006) concluíram que tratamentos a quente podem gerar o colapso dos vasos e fibras, o que, consequentemente, afeta a continuidade da propagação da onda ultrassônica.

Dessa forma, verifica-se que fatores como as altas temperaturas empregadas no processo de termorretificação, além de causar variações físicas (KARTAL et al., 2007; ESTEVES et al., 2007), mecânicas (ESTEVES \& PEREIRA, 2009), químicas (INARI et al. 2007), anatômicas (BOONSTRA et al., 2006), superficiais (HUANG et al., 2012) e 
Tabela 3 - Valores máximos, mínimos e médios dos módulos de elasticidade estático e dinâmico dos corpos de prova de Pinus taeda, submetidos a diferentes tratamentos.

\begin{tabular}{llllll}
\hline Tratamento & Propriedade & Máximo & Mínimo & Média (MPa) & CV (\%) \\
\hline \multirow{2}{*}{ Combinação } & Ec0 & 15895,3 & 8047,19 & $10526,7 \mathrm{a}$ & 15,76 \\
& Ed & 10554,1 & 7656,6 & $9198,62 \mathrm{~b}$ & 8,84 \\
\multirow{2}{*}{ Estufa } & Ec0 & 12509 & 6648,41 & $9975,38 \mathrm{a}$ & 13,27 \\
\multirow{2}{*}{ Testemunha } & Ed & 10404,2 & 8165,62 & $9068,22 \mathrm{~b}$ & 7,59 \\
& Ec0 & 11647,6 & 6879,94 & $9578,59 \mathrm{a}$ & 12,71 \\
\hline
\end{tabular}

Em que: Ec0 = Módulo de elasticidade estático obtido pelo ensaio de flexão estática (MPa), Ed= módulo de elasticidade dinâmico (MPa) e $\mathrm{CV}$ - coeficiente de variação em (\%). Entre as propriedades, em cada tratamento, médias seguidas pela mesma letra na coluna não possuem diferença estatisticamente significativa em nível de $95 \%$ de confiança, segundo teste LSD de Fisher.

colorimétricas (ESTEVES et al., 2008a), causam alterações acústicas na madeira, em que baseiam-se os fundamentos do método não destrutivo ultrassônico. Essas alterações foram influentes nas determinações do Ec0 com o Ed, em que os menores coeficientes de determinação, 0,514 e 0,476, foram encontrados para os corpos de prova que sofreram tratamento Combinado e Estufa, respectivamente.

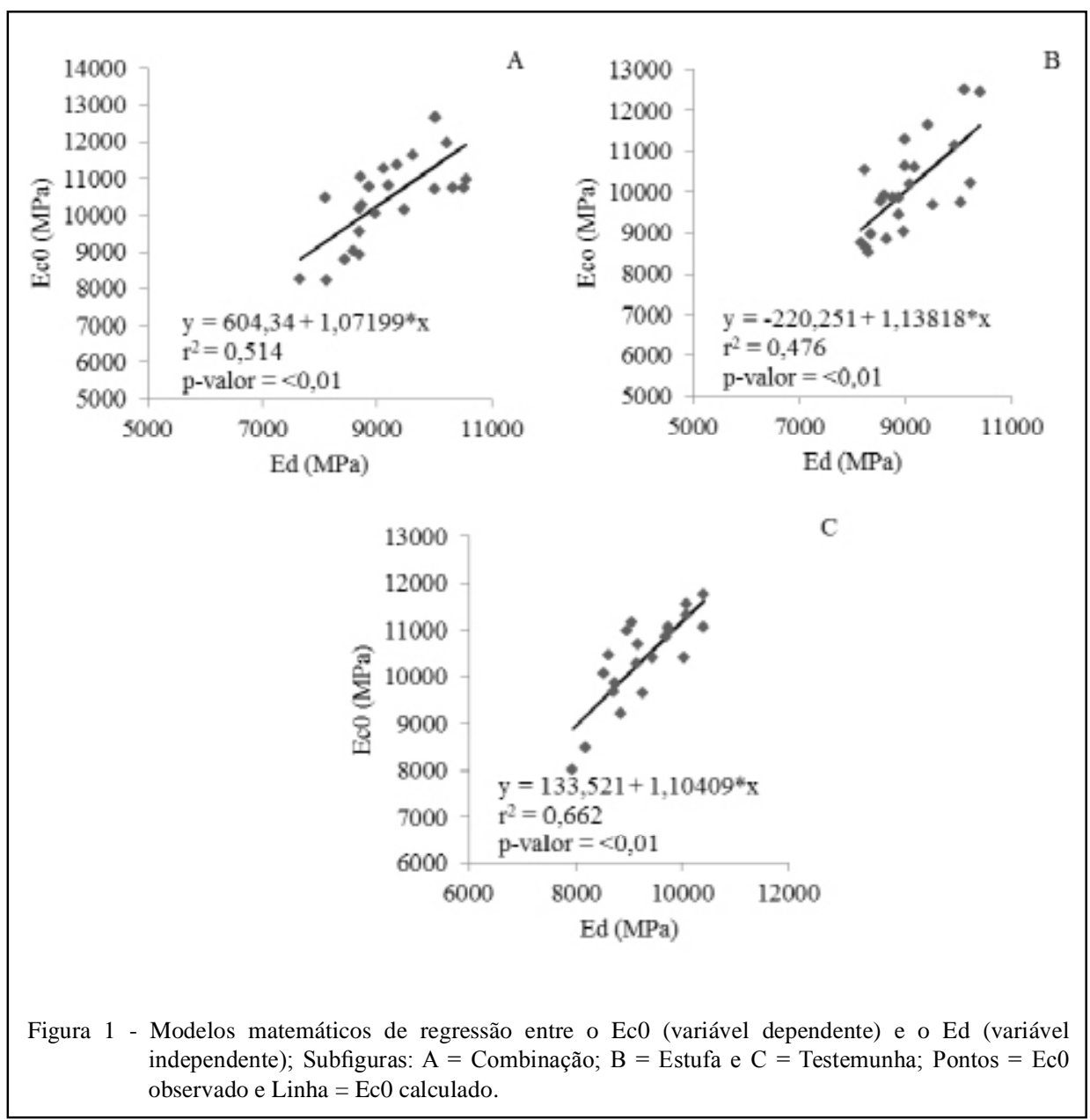

Ciência Rural, v.43, n.4, abr 2013. 
Assim, o aprimoramento e a quantificação de outros fatores influentes nos processos de termorretificação, como temperatura e tempo do processo, devem ser avaliados em estudos futuros, a fim de determinar com mais clareza e precisão o Ec0 através do Ed.

\section{CONCLUSÃO}

O módulo de elasticidade dinâmico foi bom estimador do módulo de elasticidade estático da madeira de Pinus taeda, em que todos os modelos matemáticos de regressão foram significativos em $1 \%$ de probabilidade de erro.

\section{REFERÊNCIAS}

AMERICAN SOCIETY FOR TESTING AND MATERIALS (ASTM). Standard methods of testing small clear specimens of timber: ASTM D 143-94. Philadelphia, 2000. 31p.

ASSOCIAÇÃO BRASILEIRA DE NORMAS TÉCNICAS (ABNT). Projetos de estrutura de madeira: NBR 7190. Rio de Janeiro, 1997. 107p.

BALLARIN, A.W.; NOGUEIRA, M. Determinação do módulo de elasticidade da madeira juvenil e adulta de Pinus taeda por ultrasom. Engenharia Agrícola, v.25, n.1, p.19-28, 2005. Disponível em: 〈http://www.scielo.br/pdf/eagri/v25n1/24867.pdf〉. Acesso em: 12 set. 2010. doi: 10.1590/S0100-69162005000100003.

BARTHOLOMEU, A. Classificação de peças estruturais de madeira através do ultra-som. 2001. 105f. Tese (Doutorado em Engenharia Agrícola) - Faculdade de Engenharia Agrícola, Universidade de Campinas, SP.

BOONSTRA, M.J. et al. Microstructural and physical aspects of heat treated wood. Part 2. Hardwoods. Maderas Ciencia y tecnologia, v.8, n.3, p.209-217, 2006. Disponível em: <http:// www.scielo.cl/pdf/maderas/v8n3/art07.pdf >. Acesso em: 17 out. 2012. doi: $10.4067 / \mathrm{S} 0718-221 \mathrm{X} 2006000300007$.

BRASHAW, B.K. et al. Nondestructive testing and evaluation of wood: A worldwide research update. Forest Products Journal. v. 59, n.3, p.7-14, 2009.

BUCUR, V.; BÖHNKE, I. Factors affecting ultrasonic measurements in solid wood. Ultrasonics, v.32, n.5, p.385-390, 1994. Disponível em: <http://www.sciencedirect.com/science/ article/pii/0041624X94901090\#>. Acesso em: 9 mar. 2011. doi: 10.1016/0041-624X(94)90109-0.

CALEGARI, L. et al. Monitoramento do teor de umidade de madeiras de Pinus elliottii Engelm. e Eucalyptus grandis Hill ex Maiden, sob diferentes temperaturas de secagem, através do ultrasom. Ciência Florestal, v.17, n.4, p.399-408, 2007.

CALEGARI, L. Uso da onda ultra-sônica como meio de controle do processo de secagem da madeira. 2006. 91f. Dissertação (Mestrado em Engenharia Florestal) - Curso de Pós-graduação em Engenharia Florestal, Universidade Federal de Santa Maria, RS.
ESTEVES, B. et al. Influence of steam heating on the properties of pine (Pinus pinaster) and eucalypt (Eucalyptus globulus) wood. Wood Science and Technology, v.41, n.3, p.193-207, 2007. Disponível em: <http://www.springerlink.com/content/ ym146014263m6413/fulltext.pdf $>$. Acesso em: 15 out. 2012. doi: 10.1007/s00226-006-0099-0.

ESTEVES, B. et al. Heat-induced colour changes of pine (Pinus pinaster) and eucalypt (Eucalyptus globulus) wood. Wood Science and Technology, v.42, n.5, p.369-384, 2008a. Disponível em: <http://www.springerlink.com/content/e1j508p0g4041576/fulltext. pdf>. Acesso em: 15 out. 2012. doi: 10.1007/s00226-007-0157-2.

ESTEVES, B. et al. Pine wood modification by heat treatment in air. Bioresources, v.3, n.1, p.142-154, 2008b. Disponível em: <http:// ncsu.edu/bioresources/BioRes_03/BioRes_03_1_0142_Esteves_ DP_PineWoodMod_HeatAir.pdf $>$. Acesso em: 10 mar. 2011.

ESTEVES, B.; PEREIRA, H.M. Wood modification by heat treatment: a review. Bioresources, v.4, n.1, p.370-404, 2009. Disponível em: <http://ncsu.edu/bioresources/BioRes_04/ BioRes_04_1_0370_Esteves_P_Wood_Mod_Heat_Treatment_ Rev_367.pdf>. Acesso em: 12 out. 2012.

FEENEY, F.E. et al. The influence of in homogeneity on the propagation of ultrasound in wood. Ultrasonics, v.36, n.1-5, p.449-453, 1998. Disponível em: <http://www.sciencedirect.com/ science/article/pii/S0041624X97000668>. Acesso em: 15 fev. 2012. doi: 10.1016/S0041-624X(97)00066-8.

GARCIA, R.A. et al. Nondestructive evaluation of heat-treated Eucalyptus grandis Hill ex Maiden wood using stress wave method. Wood Science and Technology, v.46, n.1-3, p.4152, 2012. Disponível em: <http://www.springerlink.com/ content/402568g72687wg47/fulltext.pdf $>$. Acesso em: 15 out. 2012. doi: $10.1007 / \mathrm{s} 00226-010-0387-6$.

HUANG, X. et al. Effect of surface preparation on the wettability of heat-treated jack pine wood surface by different liquids. European Journal of Wood and Wood Products, v.70, n.5, p.711-717, 2012. Disponível em: <http://download.springer. com/static/pdf/978/art\%253A10.1007\%252Fs00107-012-0605-z. pdf?auth66=1352651805_f0d18778a068c619485bf1836b1cfcd5\&e $\mathrm{xt}=$.pdf $>$. Acesso em: 15 out. 2012. doi: 10.1007/s00107-012-0605-z.

INARI, G.N. et al. Chemical reactivity of heat-treated wood. Wood Science and Technology, v.41, n.2, p.157-168, 2007. Disponível em: $<\mathrm{http}: / /$ www.springerlink.com/content/1p88285583g5310q/fulltext. pdf>. Acesso em: 15 out. 2012. doi: 10.1007/s00226-006-0092-7.

KARTAL, S.N. et al. Water absorption of boron-treated and heatmodified wood. Journal of Wood Science, v.53, n.5, p.454-457, 2007. Disponível em: <http://www.springerlink.com/content/ el5781852378t486/fulltext.pdf $>$. Acesso em: 15 out. 2012. doi: $10.1007 /$ s 10086-007-0877-9.

NESVIJSKI, E.G. On design of ultrasonic transducers and accuracy of velocity measurements. e-Journal of Nondestructive Testing, v.5, n.2, n.p., 2003. Disponível em: <http://www.ndt.net/ article/v05n02/nesvi/nesvi.htm>. Acesso em: 13 jan. 2011.

OUIS, D. Dispersion of wood as a consequence of its viscoelasticity. In: INTERNATIONAL SYMPOSIUM ON NONDESTRUCTIVE TESTING OF WOOD, 13., 2002, Berkeley. Proceedings... Berkeley: University of California, 2002. 8p. 
PUCCINI, C.T. Avaliação de aspectos de qualidade da madeira utilizando o ultra-som. 2002. 139f. Tese (Doutorado em Engenharia Agrícola) - Programa de Pós-graduação em Engenharia Agrícola, Universidade Estadual de Campinas, SP.

RODRIGUES, T.O. Efeitos da torrefação no condicionamento de biomassa para fins energéticos. 2009. 71f. Dissertação (Mestrado em Ciências Florestais) - Universidade de Brasília, DF

ROSS, R.J. et al. Nondestructive evaluation of wood. Forest Products Journal, v.18, n.1, p.14-19, 1998

STANGERLIN, D.M. et al. Obtenção do módulo de elasticidade em madeiras de Patagonula americana e Araucaria angustifolia por meio do método ultra-sonoro. Revista Científica Eletrônica de Engenharia Florestal, v.11, n.2, p.1-15, 2008a.

STANGERLIN, D.M. et al. Determinação do módulo de elasticidade em madeiras por meio de métodos destrutivo e nãodestrutivo. Revista Brasileira de Ciências Agrárias, v.3, n.2, p.145-150, 2008b. Disponível em: <http://132.248.9.1:8991/ hevila/AgrariaRecife/2008/vol3/no2/8.pdf>. Acesso em: 11 mar. 2011. doi: 10.5039/agraria.v3i2a284.

WANG, X. et al. Acoustic assessment of wood quality of raw forest materials - a path to increased profitability. Forest Products Journal, v.57, n.5, p.6-14, 2007.

Ciência Rural, v.43, n.4, abr 2013. 